\title{
IMPROVED SCHEME FOR CLUSTER BASED FAULT TOLERANT DATA AGGREGATION IN WIRELESS SENSOR NETWORKS
}

\author{
T. Suriya Praba ${ }^{1}$, Venkatesh Veeramuthu ${ }^{2}$, R.D. Harshitha ${ }^{3}$ and T. Sethukarasi ${ }^{4}$ \\ ${ }^{l}$ School of Computing, Shanmugha Arts, Science, Technology and Research Academy, India \\ ${ }^{2}$ Department of Computer Science and Engineering, RMK Engineering College, India \\ ${ }^{3,4}$ Department of Electronics and Communication Engineering, PET Engineering College, India
}

\begin{abstract}
Wireless Sensor Networks (WSNs) are used widely in many mission critical applications like battlefield surveillance, environmental monitoring, forest fire monitoring etc. A lot of research is being done to reduce the energy consumption, enhance the network lifetime and fault tolerance capability of WSNs. In this paper, we propose an energy aware routing algorithm for cluster based WSNs along with an ANFIS estimator based data aggregation scheme called Neuro-Fuzzy Optimization Model (ANFIS-NFO) for the design of fault-tolerant. The algorithm is based on a clever strategy of cluster head (CH) selection, residual energy of the $\mathrm{CHs}$ and the intra-cluster distance for cluster formation. To facilitate data routing, a directed virtual backbone of $\mathrm{CHs}$ is constructed which is rooted at the sink. The proposed algorithm is also shown to balance energy consumption of the CHs during data routing process. We prove that the algorithm achieves constant message and linear time complexity and they pro-actively identify the faulty $\mathrm{CH}$ s by the application of the proposed ANFIS estimator and perform inter-cluster fault tolerant data aggregation.
\end{abstract}

\section{Keywords:}

Wireless Sensor Networks, Neuro-Fuzzy Optimization Model, FaultTolerant, ANFIS

\section{INTRODUCTION}

Wireless sensor networks (WSNs) have gained enormous attention for their usage in monitoring environment, security surveillance, heath and underground mines [1]. However, the main limitation of WSNs is that the sensor nodes are operated on limited power sources. Moreover, in several applications such as in battlefields, dense forest etc. sensor nodes are not easily accessible due to hostile nature of such environment and therefore they cannot be recharged. Thus energy conservation of the sensor nodes to maximize the network lifetime is one of the most challenging issues in WSNs. Therefore, a lot of research has been carried out for energy saving of the sensor nodes for the long run operation of the WSNs.

One of the techniques to save the energy consumption is clustering sensor nodes [2]-[14]. In clustering process, sensors nodes are organized into distinct groups, called clusters and each cluster has a coordinator referred as cluster head $(\mathrm{CH})$ and remaining nodes within a cluster act as cluster members (CMs). Each sensor node must belong to one and only one cluster. Sensor nodes send their sensed data to their corresponding $\mathrm{CHs}$. $\mathrm{CHs}$ then aggregate them and send it to a remote base station called sink using single hop or multi-hop communication. Many clusterbased multi-hop routing algorithms have been developed in the literature which can be found in [15]-[22]. In most of these techniques, periodic re-clustering is performed to balance the energy consumption of the CHs. However, in such routing techniques, all neighbor $\mathrm{CHs}$ may route their data packets to a single $\mathrm{CH}$ which may deplete its energy quickly. As a result, the whole network may get partitioned in the very early stage. In addition to this, clustering algorithm also influences the performance of the routing algorithm owing to in-efficient $\mathrm{CH}$ selection, uneven $\mathrm{CH}$ distribution and ineffective cluster formation.

A sensor node cannot sustain as a $\mathrm{CH}$ if its residual energy is very low since the $\mathrm{CHs}$ are burdened with extra work as compared to their member sensor nodes. If the selected $\mathrm{CHs}$ are not well distributed in the network, then the distance between the $\mathrm{CHs}$ and their member sensor nodes is not minimal. This consumes more energy for intra-cluster communication. Furthermore, inappropriate selection by a sensor node to join a $\mathrm{CH}$ also leads to improper energy utilization.

The Non-Cluster Head sensor nodes (NCHs) sense the environment for some phenomenon of interest, collect data and forward them to their Cluster Heads $(\mathrm{CHs})$. The $\mathrm{CH}$ node performs in-network data aggregation and then forwards the aggregated data to the base station through single or multiple hops depending upon the network topology. The WSN is used widely in many applications which include search and rescue operations, battlefield surveillance, environmental monitoring, forest fire monitoring, and home automation and weather monitoring.

Generally, the WSNs are designed to operate in harsh environments with the minimum human intervention. A sensor node has to rely on its limited battery power due to the limited resources in WSNs. There are many factors that may cause the failure of a WSN. A WSN may fail due to the malfunctioning of some of its components that may be hardware or software faults, faults in the network communication layer or the application layer or may be due to battery depletion etc. The fault tolerance refers to the ability of a system to perform at a desired level even in the presence of faults. A number of research papers have been published in the area of fault detection and recovery in WSNs. But most of these mechanisms consume lots of extra energy for fault detection and recovery and even some require additional hardware and software resources for the same. The problem arises when a cluster has a large number of faulty $\mathrm{NCH}$ nodes that transmit their faulty data to the $\mathrm{CH}$ and finally to the base station. This makes the entire network unreliable for future data transmission and in severe cases, the network may collapse.

An ANFIS estimator based data aggregation scheme called Neuro-Fuzzy Optimization Model (NFOM) for fault tolerant data aggregation in WSNs. The proposed scheme employs an Adaptive Neuro-Fuzzy Inference System (ANFIS) estimator for intracluster and inter-cluster fault detection for different fault cases. The ANFIS estimator takes some fuzzy inputs from the sensor node parameters and generates a fuzzy output by the application of fuzzy rules. The generated fuzzy output is then fed to a defuzzifier which returns a crisp output about the sensor node 
status. The $\mathrm{CH}$ acts as the intra-cluster fault detection and data aggregation manager. It identifies the faulty $\mathrm{NCH}$ nodes in a cluster by the application of the proposed ANFIS estimator. The $\mathrm{CH}$ then aggregates data from only the normal $\mathrm{NCHs}$ in that cluster and forwards it to the high-energy gateway nodes. The gateway nodes act as the inter-cluster fault detection and data aggregation manager. They identify the faulty $\mathrm{CHs}$ by the application of the proposed ANFIS estimator. The faulty cluster is then isolated and not allowed to participate in the data aggregation process. This pro-active approach prevents the network from partitioning into disjoint segments due to faulty clusters. The faulty $\mathrm{CH}$ is then replaced by its nearest one-hop neighbor with maximum residual energy.

\section{RELATED WORKS}

Many clustering algorithms have been developed for WSNs. LEACH (low energy adaptive clustering hierarchy) [3] is a wellknown distributed clustering algorithm in which $\mathrm{CHs}$ are selected with some probability and remaining nodes join the nearest $\mathrm{CH}$ without considering its residual energy. Although the role of $\mathrm{CH}$ is rotated among the nodes, the overall energy consumption of nodes is neither balanced nor minimized. Moreover, this approach does not ensure even distribution of the $\mathrm{CHs}$ across the whole network. HEED (a hybrid energy-efficient distributed clustering) [4] is another popular distributed clustering algorithm that selects $\mathrm{CHs}$ by considering residual energy of the sensor nodes and intracluster distance as the primary and secondary criteria respectively. It achieves well distribution of $\mathrm{CHs}$ and minimizes intra-cluster communication cost. However, HEED introduces extra communication overhead to compute the communication cost with its neighbors by exchanging large number of messages. Many other clustering algorithms have been proposed in the literature [5]-[14]. All these algorithms introduce high message complexity in selecting $\mathrm{CHs}$ and forming the clusters, almost similar to HEED.

Multi-hop based data transmission has been considered as an efficient technique to conserve the energy of the sensor nodes. Some of the proposed techniques can be found in [15]-[22]. In CPEQ (cluster-based, periodic, event driven, and query processing) [15], a $\mathrm{CH}$ sends its data to the sink via minimum number of intermediate sensor nodes. To find the minimum hops, the sink starts the restricted flooding mechanism to configure all the nodes into number of hops away from the sink. Then a $\mathrm{CH}$ forwards the data to the next hop $\mathrm{CH}$ which is closest to the sink. In EEPA [18], a CH floods the route request packet to the sink. Upon receiving multiple copies of the packet via different paths, the sink computes the total cost of each path, a cost is embedding by each node along the path. The cost depends on the residual energy of the $\mathrm{CHs}$ along the routing path and communication energy consumed by these nodes. Then, the sink sends back the route reply message in the same reverse paths. The message contains the total cost of the path. Upon receiving the multiple copies of the route reply message, the $\mathrm{CH}$ chooses one of the paths with minimum cost and confirms the route. However, this technique constructs efficient route at the cost of huge control messages and multiple paths are constructed without use of most of them. Other approaches as reported in [19]-[22] try to build routing tree for data transmission and switch to different tree structures to alleviate the imbalance energy consumption of the sensor nodes along the routing path. Recently, an energy-aware routing algorithm called EADC has been proposed in [23]. In this algorithm, a $\mathrm{CH}$ is selected on the basis of the ratio between its residual energy to the average residual energy of its neighbors. To form clusters, each node chooses the nearest $\mathrm{CH}$ without considering its residual energy. To route the data to the sink, a $\mathrm{CH}$ chooses the next hop $\mathrm{CH}$ by considering its residual energy and load i.e., number of CMs. However, one common problem in all these techniques is that they do not assure that the relaying load of the $\mathrm{CHs}$ is balanced with respect to their residual energy. In other words, all the $\mathrm{CHs}$ are not participating in relaying the data of other $\mathrm{CHs}$ resulting imbalance of energy consumption of the CHs. Hence, it limits the network lifetime. The authors of this paper proposed an energy aware multilevel routing algorithm for cluster based WSNs, called EMRA [25]. However, the algorithm suffers from high message complexity. We also presented a clustering algorithm called BDCP [14]; but this was without any routing algorithm. However, the algorithm was experimented by assuming single hop communication between the $\mathrm{CHs}$ and the sink similar to LEACH [3]. In the present version, we extend the work of BDCP by developing a new multi-hop routing algorithm. We also incorporate a cluster formation technique which is different and more efficient than the BDCP. The advantages of the proposed algorithm are summarized as follows:

- Each sensor node independently decides its candidature for $\mathrm{CH}$ selection. Therefore, this technique does not require exchange of any control messages.

- For efficient formation of clusters, each node decides itself to join a $\mathrm{CH}$ by considering both the residual energy of the $\mathrm{CHs}$ and the distance. This results in energy saving of the WSNs.

- To balance the forwarding load of the $\mathrm{CHs}$, we devise a simple and elegant method by which each $\mathrm{CH}$ distributes and transmits the data.

\section{SYSTEM MODEL}

Here we present some assumptions for the network model used in the proposed algorithm followed by the energy model of the sensor nodes.

\subsection{WSN MODEL}

We assume that a homogenous set of sensor nodes are deployed in the target area. All the sensor nodes become static once they are deployed and the target area is completely covered by them. The sink is also static and located outside the target area. We assume that all the sensor nodes are initially provisioned with equal amount of energy. Each sensor node has given a unique identification number, sensing range, denoted by $r$, and communication range $R$ where $R=2 r$, as shown. We also assume that the inter-cluster transmission range between the $\mathrm{CHs}$ can be adjusted to $k_{R}(k=2,3$..). The maximum value of $\mathrm{k}$ is derived in the next section. The sensor nodes are aware of their locations through some localization techniques such as proposed in [24].

\subsection{WSN NETWORK MODEL}

A typical $3 \times 3$ grid cluster WSN is used as cluster model, the $\mathrm{CH}$ is located at the center with 8 number of $\mathrm{NCH}$ nodes in the 
periphery. This arrangement makes it easy for the $\mathrm{CH}$ to monitor the status of the $\mathrm{NCH}$ nodes. This $3 \times 3$ arrangement of grid cluster also facilitates easy rotation of $\mathrm{CHs}$ for load balancing. Also, the $\mathrm{CH}$ is just one-hop from all the $\mathrm{NCH}$ nodes in a $3 \times 3$ grid cluster. This feature greatly saves on energy and communication overhead. The WSN net- work model consists of a heterogeneous Wireless Sensor Network in which the nodes have varying energy.

In the WSN model, the WSN is assumed to have thousands of sensor nodes with varying energy which are grouped into a number of $3 \times 3$ grid clusters. The sensor nodes with comparatively low energy form the $\mathrm{NCH}$ node. In a cluster, the $\mathrm{NCH}$ with the highest energy becomes the $\mathrm{CH}$. The $\mathrm{CHs}$ are rotated between different NCHs as per the TDMA schedule in order to conserve energy. The base station is assumed to be stationary. Number of $3 \times 3$ grid clusters with the $\mathrm{CH}$ at the center and $\mathrm{NCHs}$ in the periphery. A record table is maintained which records the history of all local decisions during the data aggregation process. The cluster heads are selected with a probability equal to the ratio of the expected number of $\mathrm{CHs}$ to the total number of sensor nodes in the network.

\subsection{PROPOSED FAULT MODEL}

In the proposed fault model, the fault tolerance capability of WSN is assessed by observing the network's reaction to different types of faults. In the proposed work, the $\mathrm{NCH}$ and $\mathrm{CH}$ faults are injected into the network at different rates in order to simulate different fault cases. The proposed ANFIS-NFO data aggregation scheme is tested for its energy cost and network life time against sensor node fault probability and the different performance metrics are recorded. It is assumed that the fusion center or the data aggregator is unaware about the sensor fault types in advance. Depending on the type of data collected by a sensor node, the sensor faults are categorized into the following broad types.

- Fixed faults: The sensors with this kind of fault collect data with the same readings. These data are not affected by the environment. They are of two types stuck at zero faults and stuck-at-one faults. In the former, a faulty sensor node always transmits a fixed local decision ' 0 ' to the fusion center irrespective of the real observation while in the later, it always transmits a fixed local decision ' 1 ' to the fusion center irrespective of the real observation.

- Random faults: In this case, a faulty sensor node reports randomly its local decision to the fusion center irrespective of the real observation. The sensor node readings are random and uncertain.

- Transient faults: These faults may occur due to hardware features or effects of environment in the process of data collection. They can be easily corrected using the majority voting technique.

- Mixed faults: It is a combination of two or more fault types. Proposed ANFIS-NFO is pictorially represented in Fig.1.

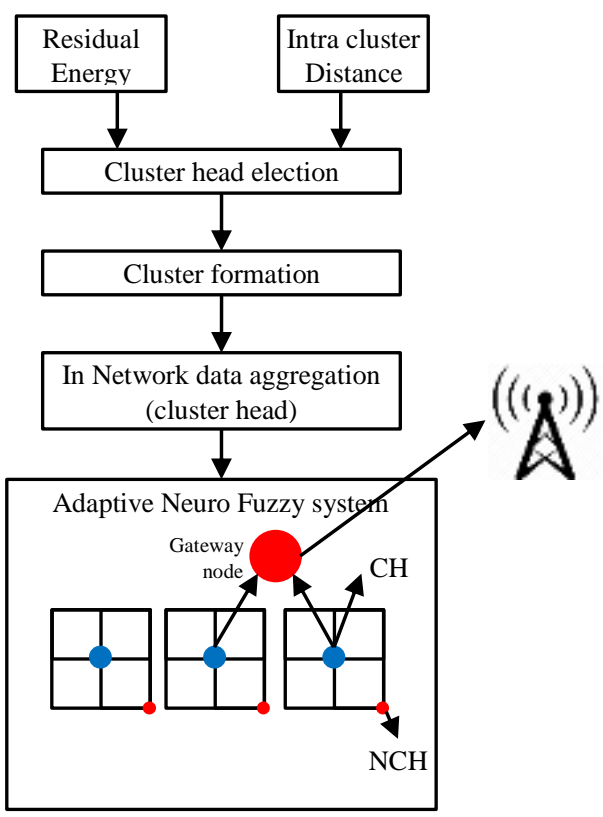

Fig.1. ANFIS-NFO

\section{PROPOSED SYSTEM}

The algorithm consists of two phases, namely clustering and fault detection. They are subsequently described in the following sections.

\subsection{CLUSTERING ALGORITHM}

\section{Algorithm I: /*CH Selection*/}

1. For each node $i$

2. $t_{i}=\left(\left(E_{m}(i)-E_{r}(i) / E_{m}(i)\right) * T_{c h}\right.$

3. end for

4. if $(t(i)==0)$ then

5. node $i$ broadcast $(\mathrm{CH} a d v t)$ in the range $\mathrm{R}$

6. end if

7. if (node $j$ receives $\mathrm{CH}$ advt.msg) then

8. node $j$ switches off its timer and becomes non- $\mathrm{CH}$ node

9. updates $N_{C H}(j)$

10. end if

11. if $(t(j)$ is non- $\mathrm{CH}$ node and receives $\mathrm{CH}$ advt.msg) then

12. updates $N_{C H}(j)$

13. end if

\section{/* Cluster Formation*/}

1. For each non-CH node $j$

2. Sum $=0.0$

3. For each $\mathrm{CH} \mathrm{k}$ belongs to $\mathrm{NCH}(\mathrm{j})$

4. Sum $=\operatorname{Sum}+E_{r}(k)$

5. end for

6. $\mu(j)=\operatorname{sum} / \mathrm{NCH}(j)$

7. for each $\mathrm{CH}_{k}$ belongs to $\mathrm{NCH}(j)$

8. if $\left(E_{r}(k) \geq \mu\right.$ and $D(j, k)<M I N$ then

9. $M I N=D(j, k)$

10. $Q=k$

11. end if

12. end for 
13. $C_{\text {set }}(Q)=\operatorname{union}\left(C_{\text {set }}(Q), j\right)$

14. end for

\section{/* Directed Virtual Backbone Formation*/}

1. Initially sink broadcasts a RREQ msg in the range $2 R$

2. $L(\sin \mathrm{k})=0$;

3. for each $\mathrm{CH}_{u}$

4. If $D(\sin k, u)<3 R)$ then

5. $P N(u)=\sin k$

6. $L(u)=L(\sin k)+1$

7. end if

8. end for

9. Recursively Node $u$ broadcast RREQ $m s g$, in the range $3 R$

10. If (node $v$ receives the $m s g$ and $L(v)>L(u)$ ) then

11. $P N(v)=u$

12. $L(v)=L(u)+1$

13. end if

Recursively, step 8 to 14 continues till all cluster heads are assigned with various levels

\subsection{FAULT DETECTION ALGORITHM}

\section{Algorithm II: Fault Detection Algorithm}

Input: Network configuration, number of simulation rounds ' $R$ ', cluster threshold CT, grid size $g d \_s i z e$, total number of clusters ' $n$ '.

Output: A fault tolerant data aggregation NFOM scheme.

for //for each simulation round

Step 1: Initialize the network parameters. Initialize node 0 as BS and node $g$ at level ' $t$ ' as the gateway node. Set count $\left[c_{k}\right]$ $=0$ for cluster $k=1,2, \ldots, n$.

Step 2: Apply the ANFIS estimator to estimate the node status of all the nodes in a cluster.

for $\forall$ cluster $C_{k}(k=1,2, \ldots, n)$

for $\forall$ node $n[i, j]$ in cluster $C_{k}$

Step 3: Apply the ANFIS estimator to estimate the node status $\mathrm{NS}(n[i, j])$ using fuzzy rules

Set the Cluster Fault Matrix (CFM) entries and forward flag ( $f w d \_f l a g$ ) values for all the nodes in a cluster.

for $\forall$ cluster $C_{k}(k=1,2, \ldots, n)$

for $\forall$ node $n[i, j]$ in cluster $C_{k}$

if $(\mathrm{NS}(n[i, j])==$ ' $\mathrm{F}$ ')

then $\operatorname{CFM}(n[i, j])=1 ; / /$ faulty node

and $f w d \_f l a g(n[i, j])=$ ' $N$ ';

// faulty $\mathrm{NCH}$ data not forwarded to $\mathrm{CH}$

else if $(N S(n[i, j])==$ ' $N$ ' $)$

then $\operatorname{CFM}(n[i, j])=0 ; / /$ normal node

and $f w d \_f l a g(n[i, j])=$ ' $Y$ ';

// normal $\mathrm{NCH}$ data forwarded to $\mathrm{CH}$

Step 4: Count the number of faulty nodes in a cluster

for $\forall$ cluster $C_{k}(k=1,2, \ldots, n)$

for $\forall$ node $n[i, j]$ in cluster $C_{k}$

if $(\operatorname{CFM}(n[i, j])==1)$

then count $\left[C_{k}\right]=\operatorname{count}\left[C_{k}\right]+1$
Step 5: Compute the Cluster Fault Index (CFI) for each cluster and forward it to the gateway node

for $\forall$ cluster $C_{k}(k=1,2, \ldots, n)$

$C F I\left[C_{k}\right]=$ count $\left.\left[C_{k}\right] * 100\right) / g d \_s i z e ;$

Step 6: Identify faulty clusters in the network by the gateway node

for $\forall$ cluster $C_{k}(k=1,2, \ldots, n)$

if $\left(\mathrm{CFI}\left[C_{k}\right]<C T\right) / /$ normal cluster

then $c f w d_{-}$flag $\left[C_{k}\right]=' Y$ '// forward data from $\mathrm{CH}$ to gateway node

else if $\left(\mathrm{CFI}\left[C_{k}\right] \geq C T\right) / /$ faulty cluster

then $c f w d \_$flag $\left[C_{k}\right]=' N$ '// faulty cluster data not forwarded

\subsection{PROPOSED CLUSTER BASED DATA AGGREGATION FAULT DETECTION SCHEME}

Here, sensor nodes are grouped into clusters as follows. Each sensor node sets its own timer independently before it starts the campaign for $\mathrm{CH}$ selection. Let $t(i)$ be the timer of sensor node $i$ which is derived as follows:

$$
t(i)=\frac{E_{m}(i)-E_{r}(i)}{E_{m}(i)} \times T_{C H}
$$

where $T_{C H}$ is the maximum allotted time for $\mathrm{CH}$ selection, $E_{m}(i)$ and $E_{r}(i)$ are the initial maximum energy and residual energy of the sensor node $i$ respectively. According to Eq.(1), a sensor node with higher residual energy will be selected as $\mathrm{CH}$ since it has shorter time. Once the timer expires then the node $i$ selects itself as a $\mathrm{CH}$ and broadcasts a $\mathrm{CH}$ announcement message in the communication range $R$. The announcement message includes its identification number (ID), residual energy $E_{r}(i)$ and location information. If a node $\mathrm{j}$ receives the message, then it withdraws its candidature for $\mathrm{CH}$ selection by cancelling its timer and becomes a non- $\mathrm{CH}$ node for the upcoming communication round. Node $\mathrm{j}$ also starts keeping track of the sensor nodes from which it receives $\mathrm{CH}$ announcement messages by maintaining a neighbor $\mathrm{CH}$ set denoted by $\mathrm{NCH}(i)$. Node $j$ decides its cluster membership in the later stage by using $\mathrm{NCH}(i)$.

To form the clusters, each non-CH node decides its cluster membership as follows. Node $j$ needs to join one of the $\mathrm{CHs}$ belonging to the set $N C H(j)$. Let $v_{1}, v_{2}, v_{3}, . ., v_{m}$ be the set of $\mathrm{CHs}$ belonging to the set $N C H(j)$. Then, the node $j$ computes the average residual energy of the $\mathrm{CHs}$, denoted by $\mu(j)$ which is calculated as follows:

$$
\mu(i)=\frac{\sum_{i=1}^{m} E_{r}\left(v_{i}\right)}{m} .
$$

Node $j$ joins the nearest $\mathrm{CH}$ whose residual energy is greater than or equal to $\mu$ by broadcasting a cluster join message in the communication range $R$. Note that the approach of $\mathrm{CH}$ selection distributes the $\mathrm{CHs}$ across the whole network evenly, i.e., no two $\mathrm{CHs}$ are neighbor to each other. The pseudo code of the algorithm is shown in Algorithm 1.

To route the data to the sink, a directed virtual backbone (DVB) of the CHs rooted at the sink is constructed as follows. 
Initially, the sink sends a route request message RREQ to the $\mathrm{CHs}$ in the range $2 R$. The message contains its ID, level $(L)$ and location information. The level of the sink is assumed to be at zero, i.e., $L(\operatorname{sink})=0$. When a $\mathrm{CH} u$ receives the message then the node increments its level to one higher than the sink, i.e., $L(u)=$ $L($ sink $)+1$ and sets the sink as its parent node $(\mathrm{PN})$, i.e., $P N(u)=$ sink. In other words, all the $\mathrm{CHs}$ within the range $3 R$ to the sink are designated as level one. Recursively, node $\mathrm{u}$ broadcasts a modified RREQ message to the $\mathrm{CHs}$ in the range $3 R$. The message consists of its ID, $L(u), E_{r}(u)$ and location information. If a $\mathrm{CH} v$ receives the message and if its level is equal or less than the level of the node $u$, then it simply discards the message. Otherwise, it updates its level to one more than the level of node $u$ and sets it as one of the PNs, i.e., $P N(v)=u$. Recursively, all the $\mathrm{CHs}$ broadcasts the RREQ to complete the process of forming DVB.

The ANFIS estimator in the proposed model monitors the status of each $\mathrm{NCH}$ node in a cluster and assigns a node status to each node by the application of fuzzy rules. The node status value may be 0 (normal) or 1 (faulty). The node status of a $3 \times 3$ grid cluster is stored in a Cluster Fault Matrix (CFM). A CFM is generated for each cluster and the Cluster Fault Index (CFI) is calculated. If the CFI value is greater than or equal to the Cluster Threshold value (CT), then the cluster is identified as a faulty one and is isolated. The faulty cluster is not allowed to participate in the data aggregation process.

The fuzzy rules are generated using a combination of four fuzzy inputs for inter and intra-cluster criteria - residual node energy (RNE), packet delivery ratio (PDR), fault ratio (FR) and number of re-transmissions (NOR) which are denoted by $u_{1}, u_{2}, u_{3}$ and $u_{4}$. The RNE is given as the energy remaining after each simulation round. The PDR is given as the ratio of the number of data packets successfully sent to the $\mathrm{CH}$ to the total number of data packets. The FR is given as the ratio of the number of simulation rounds in which the $\mathrm{NCH}$ is found to be faulty to the total number of simulation rounds. The NOR is given by the number of times the data are transmitted again (re-transmitted). The RNE values vary from Very Low (VL), Low (L) and Medium (M) to High (H) and have a trapezoidal membership function. For example, if the residual energy of a $\mathrm{NCH}$ node is $0.05 \mathrm{~J}$, it may be assigned Very Low (VL) level. A trapezoidal M is specified by four parameters. The PDR, FR and NOR values vary from Good (G), Average (A) and Poor (P) to Very Poor (VP) and have a trapezoidal membership function assigned to them. These values are updated by the $\mathrm{CH}$ at the end of each simulation round and are stored in a record table. The fuzzy output is the Node Status (NS) which can take values - Normal (N) or Faulty (F) according to the fuzzy rules and has a triangular membership function assigned. The fuzzy output NS is updated periodically to the $\mathrm{CH}$ in intra-cluster and to the gateway node in inter- cluster data aggregation process.

The job of the NCHs is to sense the environment, collect data and forward it to their respective $\mathrm{CHs}$. The $\mathrm{CH}$ acts as the intracluster fault detection and data aggregation manager. It performs three vital functions - monitor the status of each $\mathrm{NCH}$ in the grid cluster; pro-actively identify the faulty NCHs by the application of the proposed ANFIS estimator and per- form intra-cluster fault tolerant data aggregation. The aggregated data are then forwarded by the $\mathrm{CH}$ to high energy nodes called the gateway nodes. The gateway nodes act as the inter-cluster fault detection and data aggregation manager. They perform three vital functions monitor the status of each $\mathrm{CH}$; pro-actively identify the faulty $\mathrm{CH}$ s by the application of the proposed ANFIS estimator and perform intercluster fault tolerant data aggregation. The gateway nodes have higher energy than the $\mathrm{CH}$ nodes. So, they are entrusted with the additional responsibility of replacing the faulty $\mathrm{CH}$ in a cluster by its nearest one-hop NCH node with the highest residual energy. This change in $\mathrm{CH}$ is communicated to all the NCHs in the cluster and the base station and the routing table is updated accordingly. Finally, the aggregated data are forwarded from the gateway node to the base station.

\section{RESULTS AND DISCUSSIONS}

Implementation of the proposed technique clearly yields a better result when considering the cost spent for the power consumption. When the proposed method is applied the cost decreases by around $62.5 \%$ which is a really drastic improvement. Although there isn't much reduction as the node's fault probability increases, there sure is a considerable improvement when compared to the NFOM algorithm, hence one can confidentially claim that the algorithm fulfils the objective for which it was proposed. Power consumption has always been a bottle neck when we consider WSNs as already mentioned. Hence, any new algorithm's primary goal will be to reduce the power consumption by any means possible. It is always better to adopt a technique those techniques because not only will the performance be better it will also be a step toward a healthier environment in the future. The results prove the above mentioned in the Fig.2.

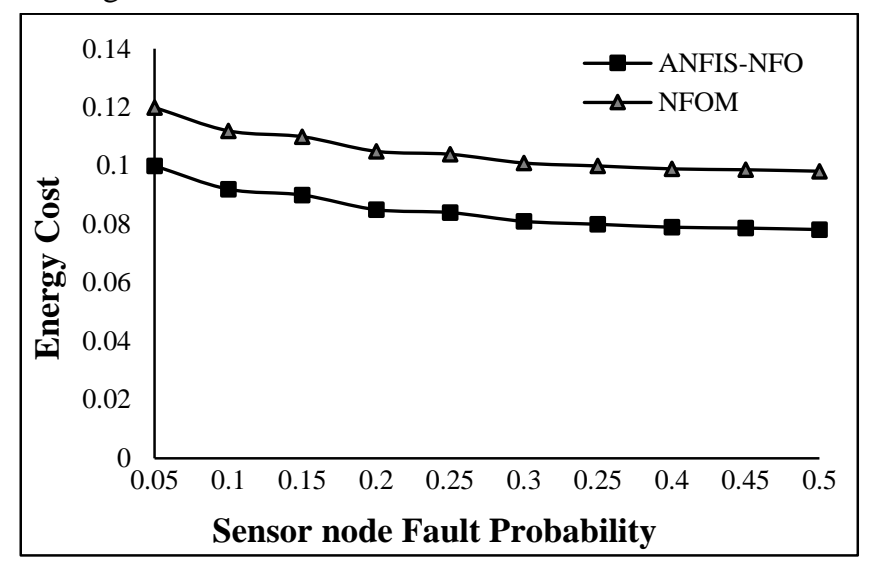

Fig.2. Energy Cost (NFOM vs ANFIS-NFO)

Implementation of the proposed technique clearly yields a better result when considering the cost spent for the power consumption. When the proposed method is applied the cost decreases by around $62.5 \%$ which is a really drastic improvement. Although there isn't much reduction as the node's fault probability increases, there sure is a considerable improvement when compared to the NFOM algorithm, hence one can confidentially claim that the algorithm fulfils the objective for which it was proposed. Power consumption has always been a bottle neck when we consider WSNs as already mentioned. Hence, any new algorithm's primary goal will be to reduce the power consumption by any means possible. It is always better to adopt a technique those techniques because not only will the performance be better it will also be a step toward a healthier environment in the future. 


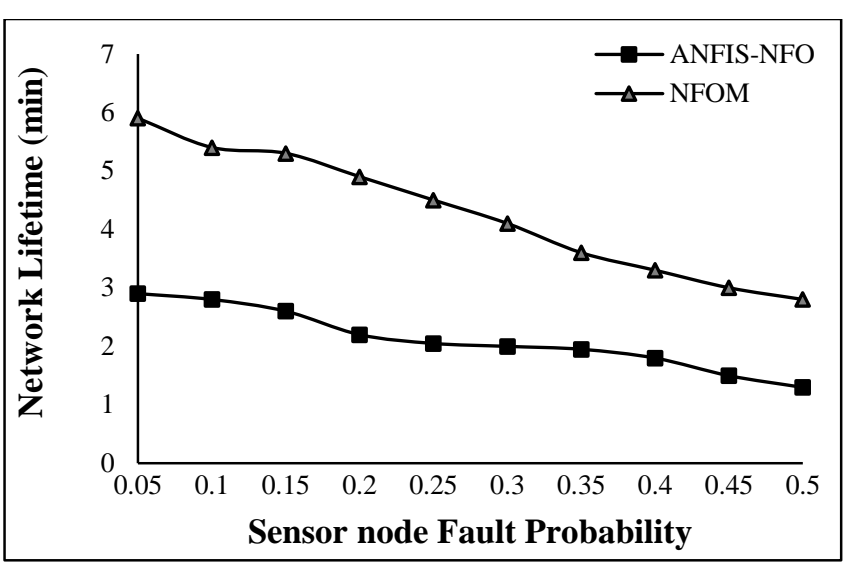

Fig.2. Network Lifetime (NFOM vs ANFIS-NFO)

The Fig. 2 compares the network lifetime of NFOM and the proposed ANFIS-NFO algorithm as the node's fault probability increases. This result is a consequence of the results discussed along with Fig.2. As the power consumption decreases the lifetime of the network increases. Since the proposed algorithm showed lesser power consumption it is predictable that the lifetime of the network increases. The difference between the two algorithms aren't as evident as the sensor node's fault probability increases but, the proposed algorithm promises a betterment always. The algorithm guarantees an improved lifetime to the network.

\section{CONCLUSIONS}

Conservation of energy is the main challenge in the development of wireless sensor networks. We have presented in this paper, a new energy efficient fault tolerant routing algorithm for wireless sensor networks. The algorithm consists of clustering and fault detection phases. We have shown that there is no requirement of any exchange of control message for cluster head selection. We have developed an efficient strategy to organize all the $\mathrm{CHs}$ into various levels for constructing a directed virtual backbone to facilitate data routing toward the sink. The proposed scheme is observed to be the best in terms of energy cost, network life- time, loss probability, fault detection accuracy and false alarm rate as compared to the other existing algorithms. The computation of the proposed algorithm for different possible positions of the base station is an interesting topic which can be taken as a future research work. Also, this work may be further extended in future for designing secure data aggregation schemes using cryptographic techniques for the design of fault tolerant WSNs.

\section{REFERENCES}

[1] Mohammad A. Haque, Ramin Irani, Kamal Nasrollahi and Thomas B. Moeslund, "Heart Beat Rate Measurement from Facial Video", IEEE Intelligent Systems, Vol. 31, No. 3, pp. 1-6, 2016.

[2] H. Rahman and M.U. Ahmed, S. Begum and P. Funk, "Real Time Heart Rate Monitoring from Facial RGB Color Video using Webcam", Proceedings of $29^{\text {th }}$ Annual Workshop of the Swedish Artificial Intelligence Society, pp. 1-8, 2016.

[3] Simmi Dutta, Hiteshwar, Abhimanyu Dev Jamwal and Azhar Ud Din Guroo, "Heart Rate Detection using Independent Component Analysis and Multivariate Adaptive Regression Splines", Imperial Journal of Interdisciplinary Research, Vol. 2, No. 10, pp. 1-7, 2016.

[4] M. Kumar, A. Veeraraghavan and A. Sabharwal, "Distance PPG: Robust Non-Contact Vital Signs Monitoring using A Camera", Biomedical Optics Express, Vol. 6, No. 5, pp. 1565-1588, 2015.

[5] Hussain A. Jaber, A.L. Ziarjawey and Ilyas Cankaya, "Heart Rate Monitoring and PQRST Detection Based on Graphical User Interface with Matlab", International Journal of Information and Electronics Engineering, Vol. 5, No. 4, pp. 311-317, 2015.

[6] J. Moreno, J. Ramos-Castro, J. Movellan, E. Parrado, G. Rodas and L. Capdevila, "Facial Video-based Photoplethysmography to Detect HRV at Rest", International Journal of Sports Medicine, Vol. 36, No. 6, pp. 474-480, 2015.

[7] Larissa Carvalho, H.G. Virani and S. Kutty, "Analysis of Heart Rate Monitoring Using a Webcam", International Journal of Advanced Research in Computer and Communication Engineering, Vol. 3, No. 5, pp. 1-7, 2014.

[8] X. Li, J. Chen, G. Zhao, and M. Pietikainen, "Remote Heart Rate Measurement from Face Videos under Realistic Situations", Proceedings of IEEE International Conference on Computer Vision and Pattern Recognition, pp. 42644271, 2014.

[9] R. Irani, K. Nasrollahi and T.B. Moeslund, "Improved Pulse Detection from Head Motions using DCT", Proceedings of $9^{\text {th }}$ International Conference on Computer Vision Theory and Applications, pp. 124-129, 2014.

[10] S. Thulasi Prasad and S. Varadarajan, "Heart Rate Detection using Hilbert Transform", International Journal of Research in Engineering and Technology, Vol. 2, No. 8, pp. 12-18, 2013.

[11] Gerard De Haan and Vincent Jeanne, "Robust Pulse Rate from Chrominance-Based rPPG", IEEE Transactions on Biomedical Engineering, Vol. 60, No. 10, pp. 94-128, 2013.

[12] G. Balakrishnan, F. Durand and J. Guttag, "Detecting Pulse from Head Motions in Video", Proceedings of IEEE International Conference on Computer Vision and Pattern Recognition, pp. 3430-3437, 2013.

[13] X. Yu, J. Huang, S. Zhang, W. Yan and D. Metaxas, "Posefree Facial Landmark Fitting Via Optimized Part Mixtures and Cascaded Deformable Shape Model", Proceedings of IEEE International Conference on Computer Vision and Pattern Recognition, pp. 1944-1951, 2013.

[14] Isayiyas Nigatu Tiba and Li Li, "Image-Based Automatic Pulse Rate Monitoring System Using PC Webcam”, International Journal of Engineering Research and Technology, Vol. 2, No. 12, pp. 841-847, 2013.

[15] M. Soleymani, J. Lichtenauer, T. Pun and M. Pantic, "A Multimodal Database for Affect Recognition and Implicit Tagging”, IEEE Transactions on Affective Computing, Vol. 3, No. 1, pp. 42-55, 2012. 